\title{
PERCEPCIÓN Y CALIDAD DE AGUA EN COMUNIDADES RURALES DEL ÁREA NATURAL PROTEGIDA LA ENCRUCIJADA, CHIAPAS, MÉXICO
}

\author{
(Perception and water quality in rural communities of the protected area La Encrucijada, Chiapas, Mexico)
}

Elba FAVIEL CORTEZ ${ }^{1}$, Dulce INFANTE MATA ${ }^{1 *}$ y Dolores O. MOLINA ROSALES ${ }^{2}$

${ }^{1}$ Departamento de Ciencias de la Sustentabilidad, El Colegio de la Frontera Sur, Unidad Tapachula, km 2.5 carretera Antiguo Aeropuerto, 30700 Tapachula, Chiapas, México

${ }^{2}$ Departamento de Ciencias de la Sustentabilidad, El Colegio de la Frontera Sur, Unidad Campeche, Av. Rancho Polígono 2-A, Ciudad Industrial, Lerma, 24500 Campeche, Campeche, México

*Autor para correspondencia: dulce.infante@gmail.com

(Recibido junio 2017; aceptado agosto 2018)

Palabras clave: zona costera, isla de barrera, orilla del estero, recurso hídrico, dunas

\section{RESUMEN}

El presente estudio muestra las percepciones de los habitantes de ocho comunidades ubicadas en el área natural protegida La Encrucijada, Chiapas, con relación a la calidad, disponibilidad y accesibilidad de agua de pozos artesianos y agua potable embotellada y entubada, así como la evaluación de la calidad del agua de 29 pozos artesianos, nueve marcas de agua potable embotellada y dos fuentes de agua entubada. La información sobre percepciones en relación con la disponibilidad y calidad del agua que consume la población se obtuvo mediante 105 entrevistas domiciliarias con preguntas cerradas. La calidad del agua se evaluó por medio de parámetros fisicoquímicos ( $\mathrm{pH}, \mathrm{NO}_{2}, \mathrm{NO}_{3}$ y alcalinidad) y bacteriológicos (coliformes fecales). En seis comunidades, los pozos artesianos son la fuente de agua segura todo el año y su uso es mayormente doméstico. En siete comunidades, el agua embotellada se ha convertido en la principal fuente para beber. Las seis comunidades que cuentan con pozo artesiano como única fuente de agua identifican mejor los meses en que escasea el agua en su pozo y perciben el cambio en el sabor del agua en comparación con las dos comunidades que tienen acceso a agua entubada. La percepción de la calidad del agua de diferentes fuentes determina el uso de ésta. De los 29 pozos artesianos evaluados, 18 rebasaron el límite permisible para $\mathrm{NO}_{3}(>10 \mathrm{mg} / \mathrm{L})$, uno para $\mathrm{NO}_{2}(>0.05 \mathrm{mg} / \mathrm{L})$, dos para alcalinidad $(>300 \mathrm{mg} / \mathrm{L})$ y en 27 hay presencia de coliformes fecales.

Key words: coastal system, barrier island, estuary shore, hydric resource, dunes

\begin{abstract}
The present study shows the perceptions of the inhabitants of eight communities located in La Encrucijada, Chiapas in relation to the quality, availability and accessibility of well water and bottled and piped water, as well as the evaluation of water quality in 29 wells, nine brands of jug water and two sources of tubed water. Information regarding the perception of the population regarding availability and quality of water for human consumption was obtained through a survey which consisted in 105 home interviews
\end{abstract}


with closed-ended questions. As for water quality analysis, physicochemical parameters ( $\mathrm{pH}, \mathrm{NO}_{2}, \mathrm{NO}_{3}$, alkalinity) and bacteriological parameters (fecal coliforms) were analyzed. Based on the home interviews, it was found that in six communities, traditional wells are the source of safe water all year round, and their use is mostly domestic. In seven communities bottled water has become the main source for drinking. The six communities that have a well as the only source of water better identify the months in which water is scarce in their well and perceive the change in the taste of water compared to the two communities that have access to piped water. The perception of water quality of diverse sources determines its use. Of the 29 wells evaluated, 18 exceeded the maximum permissible limits for $\mathrm{NO}_{3}(>10 \mathrm{mg} / \mathrm{L})$, one for $\mathrm{NO}_{2}(>0.05 \mathrm{mg} / \mathrm{L})$, two for alkalinity ( $>300 \mathrm{mg} / \mathrm{L})$ and 27 for fecal coliforms.

\section{INTRODUCCIÓN}

La problemática de acceso a fuentes de agua con la calidad y disponibilidad adecuada para abastecimiento humano es un tema de vital importancia tanto para las autoridades como para la sociedad en general (Sánchez-Pérez et al. 2000, Pérez et al. 2011, CARE 2012). En México, la cobertura nacional de agua potable es del $92.5 \%,(95.7 \%$ en zonas urbanas y $81.6 \%$ en zonas rurales). En sistemas de alcantarillado, la cobertura nacional es de $91.0 \%$ (96.6 \% en zonas urbanas y $74.2 \%$ en zonas rurales) (CONAGUA 2016). En Chiapas, el $51 \%$ de la población vive en áreas donde el acceso a servicios básicos es limitado (CONAPO 2015), lo cual ubica a este estado, de acuerdo con Kauffer (2014), entre los de menor porcentaje de acceso a agua entubada $(87.2 \%)$ y a sistemas de drenaje (86.9 \%) a nivel nacional (INEGI 2015).

Una fuente de agua que debe considerarse es el agua subterránea en las dunas costeras. Estos ecosistemas se consideran prestadores de servicios ambientales, ${ }^{1}$ especialmente en servicios de provisión (MorenoCasasola 2010). Las dunas costeras proveen agua para diversos usos, mantienen su calidad (Camacho-Valdez et al. 2013), purifican y asimilan nutrientes y herbicidas (Fisher y Acreman 2004, Cejudo-Espinosa et al. 2008), funcionan como barreras para la intrusión salina (Mollema et al. 2008) y sobre todo proveen agua para consumo humano (Everard et al. 2010).

En la zona costera de Chiapas, la mayoría de las localidades ubicadas en la franja de barrera sobre la duna utilizan pozos artesianos ${ }^{2}$ como fuente de agua para consumo (Machuca 2014). En la reserva de la biósfera de La Encrucijada, las localidades situadas en el área tienen como fuente principal de abastecimiento de agua los pozos artesianos (Liscovsky et al. 2012).

Estudios realizados en el área de la reserva han demostrado que las fuentes de agua situadas en la franja costera (entre ellos los pozos artesianos) están expuestas a diversos contaminantes, entre los que se encuentran descargas de basura, desechos sólidos y aguas residuales con excesiva carga de nutrientes provenientes de aproximadamente 17 ríos contaminados por los asentamientos urbanos aguas arriba (Gómez-Ortega 2013, Hernández 2014, Machuca 2014). En consecuencia, los pozos se ven afectados por la intrusión salina derivada de la erosión de la playa y las dunas costeras (Barrios-Ramos 2013), la cual ocasiona el aumento en la salinidad del agua (Machuca 2014). También se ha detectado variabilidad estacional en la disponibilidad de agua en los pozos, la cual generalmente es más baja en la temporada de estiaje de acuerdo con las evaluaciones de algunos estudios y de lo que percibe la población (GómezOrtega 2013, Machuca 2014). Según Liscovsky et al. (2012), en la temporada de estiaje la gente recurre a la compra de agua embotellada como fuente alterna porque percibe una disminución en la cantidad de agua en sus pozos.

Pese a que no se han realizado estudios exclusivos sobre percepción de la calidad del agua en La Encrucijada, Machuca (2014), Jones y Mach (2012), y Liscovsky (2012) lograron identificar que las personas perciben alteración en la calidad del agua de pozos y esteros que afecta directamente algunas de las actividades que realizan, tanto en el vivienda, como la disminución de la productividad pesquera.

\footnotetext{
1 "Son las condiciones y los procesos a través de los cuales los ecosistemas naturales mantienen y satisfacen la vida del ser humano" (Moreno-Casasola 2010).

2 "Los pozos artesianos se caracterizan por ser de tecnología antigua, se cavan a mano con picos y palas hasta alcanzar la tabla de agua, es decir el nivel superior de aguas subterráneas someras o aguas freáticas de un acuífero” (Chávez et al. 2013).
} 


\section{Percepción ambiental como fuente de información}

Los estudios de percepción permiten conocer las interpretaciones y los significados en torno a las impresiones obtenidas del ambiente y los efectos que tienen sobre los usuarios (Arias 2006). Calixto y Herrera (2010) mencionan que la percepción ambiental implica reconocer el ambiente a través de los sentidos; además, proporciona las bases para dirigir y regular actividades cotidianas del individuo. Fernández-Moreno (2008) considera que las percepciones ambientales son fuente de información importante para planeadores y manejadores de un territorito.

Otro aspecto importante de la percepción es que permiten profundizar la valorización subjetiva que realizan las personas con relación al significado de los servicios ecosistémicos (Álvarez-Salas et al. 2016), especialmente cuando se pretenden implementar estrategias de conservación, ya que la valorización de los servicios ambientales debe enriquecerse con el conocimiento de la población local (Carrera et al. 2016). Las distintas formas de percibir el entorno también están determinadas por diversas variables que influyen en los procesos perceptivos como la edad, el sexo, la densidad y el tamaño poblacional, la experiencia (Arias 2006, Jones y Mach 2012), el sistema de poder político (Benez 2008), el sistema educativo (Arellano et al. 2009), la religión y las actividades cotidianas (González-Figueroa et al. 2007,
Machuca 2014), y la calidad y disponibilidad de los recursos (Corral-Verdugo et al. 2008).

Por ello, el objetivo del presente estudio fue conocer las percepciones de los habitantes de ocho comunidades de La Encrucijada sobre la calidad, disponibilidad y accesibilidad de las fuentes de agua que utilizan para uso y consumo, así como determinar la calidad del agua con base en parámetros físicos, químicos y microbiológicos.

\section{MATERIALES Y MÉTODOS}

\section{Área de estudio}

La Encrucijada (sitio RAMSAR núm. 815) se localiza al sur del estado de Chiapas, en la región fisiográfica denominada Planicie Costera del Pacífico, geográficamente ubicada entre los $14^{\circ} 43^{\prime}$ y $15^{\circ} 40^{\prime}$ latitud norte y $92^{\circ} 26^{\prime}$ y $93^{\circ} 20^{\prime}$ longitud oeste. Posee una superficie de 144868 ha, de las cuales 36216 ha pertenecen a dos zonas núcleo (La Encrucijada y Palmarcito) y 108651 ha a la zona de amortiguamiento. La Encrucijada comprende parte de los municipios de Pijijiapan, Mapastepec, Acapetahua, Huixtla, Villa Comaltitlán y Mazatán (Fig. 1).

La construcción de viviendas depende principalmente de los recursos que ofrece el medio natural: paredes de palma real (Sabal mexicana), techo de

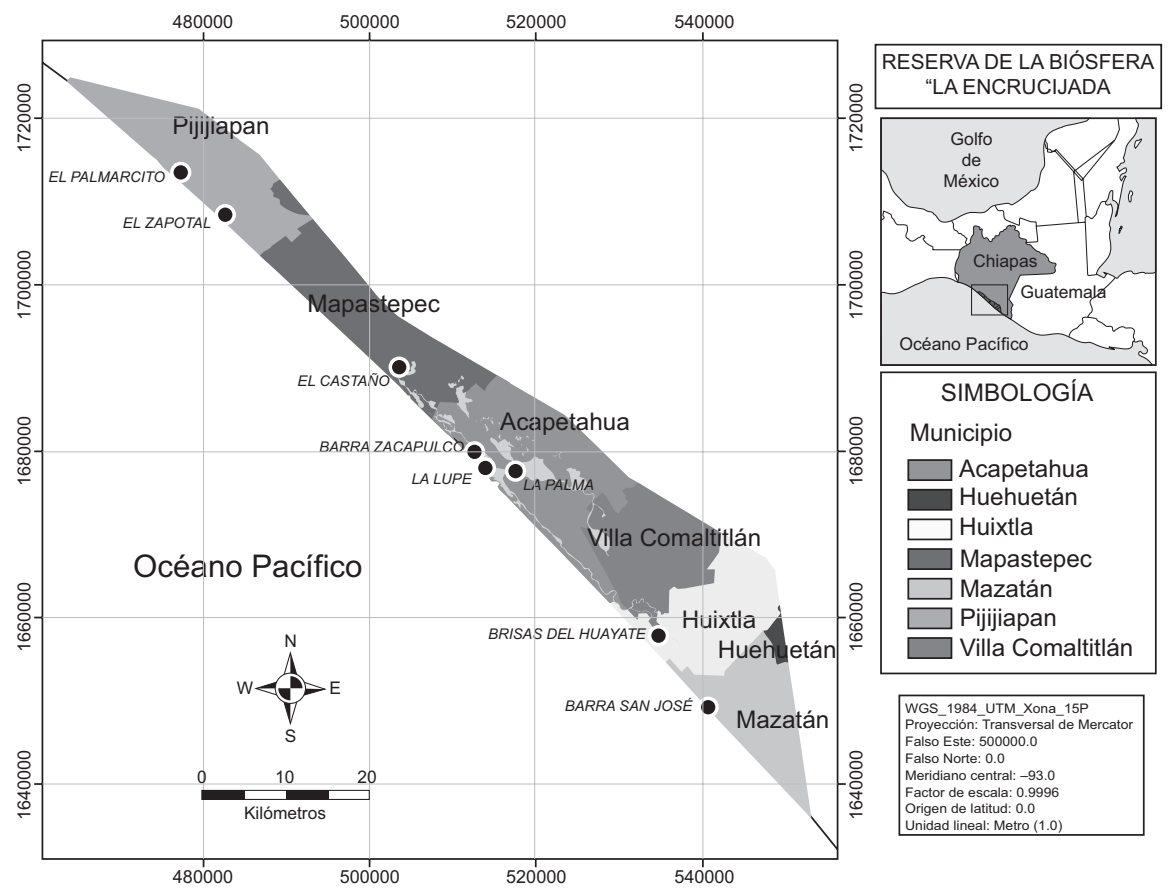

Fig. 1. Reserva de la biósfera La Encrucijada, ubicada en el suroeste del Pacífico mexicano 
palma, piso de tierra y, en algunos casos, de cemento con techo de loza o lámina. El servicio de energía eléctrica es deficiente en todas las comunidades, especialmente en la temporada de lluvias, debido a que las tormentas ocasionan el desprendimiento de ramas de mangle sobre el cableado y por ende su ruptura. Los servicios de salud y escuelas son escasos en las comunidades, por lo que la gente debe recurrir a la cabecera municipal para atender sus necesidades.

\section{Muestreo y selección de las comunidades}

Se emplearon el muestreo probabilístico y el muestreo aleatorio estratificado (Cochran 2000). Para este estudio se identificaron dos estratos, islas de barrera y orilla del estero, los cuales se eligieron en función de los servicios naturales que brindan a las comunidades. Ambos estratos poseen comunidades con menos de 1000 habitantes; incluso, algunas que no rebasan los 200 habitantes están alejadas de la cabecera municipal y se accede a ellas por carretera y vía fluvial (CONAPO 2015). En cada estrato se priorizaron las comunidades a partir de la revisión de la literatura y con base en dos criterios. El primero corresponde a la ubicación y el segundo a la principal fuente de suministro de agua (INEGI 2010). Cada estrato quedó conformado por cuatro comunidades. El primero, denominado isla de barrera, quedó constituido por las comunidades La Lupe, Brisas del
Hueyate, Barra San José y El Zapotal. El segundo, denominado orilla del estero, estuvo integrado por las comunidades El Castaño, El Palmarcito, Barra Zacapulco y La Palma.

El tamaño de la muestra (Cuadro I) se calculó de acuerdo con el muestreo probabilístico estratificado proporcional (Cochran 2000).

La unidad de análisis fueron las viviendas y el tamaño de la muestra se determinó en función del número total de viviendas con una proporción del $10 \%$. La elección de viviendas se hizo de manera aleatoria. Una vez en campo se enumeraron y se seleccionaron aleatoriamente con ayuda del programa $\mathrm{R}$ versión 3.1.2. En cada vivienda seleccionada se entrevistó a un miembro de la familia que cumpliera con dos criterios importantes: ser mayor de edad y tener aproximadamente 5 años de residir en la comunidad.

El instrumento metodológico que se utilizó fue la entrevista, que se basó en escalas de intensidad o apreciación, de distancia social con preguntas en abanico y dicotómicas, ${ }^{3}$ y de evaluación semántica diferencial $^{4}$ (Pimienta-Lastra 1999, 2000). Dichas escalas permitieron determinar niveles de preferencia y su percepción sobre la calidad del agua en la fuente identificada (buena ${ }^{5}$, regular ${ }^{6}$ y mala ${ }^{7}$ ). Las preguntas fueron cerradas con una serie de alternativas de respuesta sencillas y cortas. Para su aplicación en campo se hizo la validación de caso único, experto

CUADRO I. TAMAÑO DE MUESTRA PARA EFECTUAR LAS ENTREVISTAS EN LAS COMUNIDADES DE ESTUDIO

\begin{tabular}{|c|c|c|c|c|c|c|}
\hline Ubicación & Comunidad & $\begin{array}{l}\text { Población } \\
\text { total }\end{array}$ & Hombres & Mujeres & $\begin{array}{l}\text { Número de } \\
\text { viviendas }\end{array}$ & $\begin{array}{c}\text { Número de viviendas } \\
\text { muestreadas }\end{array}$ \\
\hline \multirow{5}{*}{$\begin{array}{l}\text { Isla de } \\
\text { barrera }\end{array}$} & La Lupe & 157 & 74 & 83 & 32 & 3 \\
\hline & El Zapotal & 929 & 447 & 482 & 208 & 21 \\
\hline & Barra San José & 756 & 391 & 365 & 205 & 20 \\
\hline & El Hueyate & 171 & 91 & 80 & 37 & 4 \\
\hline & & 2013 & 1003 & 1010 & 482 & 48 \\
\hline \multirow{5}{*}{$\begin{array}{l}\text { Orilla del } \\
\text { estero }\end{array}$} & La Palma & 678 & 281 & 291 & 173 & 17 \\
\hline & Barra Zacapulco & 399 & 209 & 190 & 102 & 10 \\
\hline & El Castaño & 75 & 29 & 46 & 16 & 2 \\
\hline & El Palmarcito & 1118 & 541 & 577 & 284 & 28 \\
\hline & & 2270 & 1060 & 1104 & 575 & 57 \\
\hline
\end{tabular}

\footnotetext{
${ }^{3}$ Con opciones de repuesta tales como: si/no o, en su caso, no sé o no responde.

${ }_{5}^{4}$ Con opciones de respuesta como: bueno, regular y malo.

${ }_{6}^{5}$ Agua sin sedimento, sin olor y sin sabor.

${ }^{6}$ No beben el agua con presencia de sedimento, mal olor, sabor a sal, pero sirve para otras actividades.

${ }^{7}$ Agua con mucho sedimento con coloración amarilla, olor a huevo podrido y sabor a sal.
} 
y piloto (Martínez-Carazo 2006) donde aplicaron 27 cuestionarios. Después de la prueba piloto se rediseñó la encuesta final con 40 preguntas (Anexo 1). Para efectos de este artículo se incluyeron únicamente las respuestas a las preguntas $1,3,4,5,6,10,13,16$, 17,21 у 22.

\section{Calidad del agua}

Para cada comunidad se muestrearon cinco puntos entre pozos, agua potable embotellada de garrafón (según el número de marcas de garrafón) y agua entubada (para las comunidades que cuentan con este servicio). En total se muestrearon 29 pozos artesianos, nueve marcas de garrafón y dos muestras de agua entubada. Los parámetros analizados fueron: oxígeno $\left(\mathrm{O}_{2}\right)$, conductividad eléctrica $(\mathrm{CE})$, salinidad (ppt), $\mathrm{pH}$, temperatura $\left({ }^{\circ} \mathrm{C}\right)$, nitratos $\left(\mathrm{NO}_{3}\right)$, nitritos $\left(\mathrm{NO}_{2}\right)$, alcalinidad y coliformes fecales. Los parámetros de temperatura, salinidad, $\mathrm{O}_{2}$ y $\mathrm{CE}$ se evaluaron in situ, utilizando el equipo multiparamétrico YSI modelo 85. El potencial de hidrógeno se analizó con un sensor de $\mathrm{pH}$ Ecotest modelo $\mathrm{pH} 2$. El método utilizado para la determinación de $\mathrm{NO}_{3}$ y $\mathrm{NO}_{2}$ correspondió a lo establecido en el manual para el equipo Hach DR/890, previamente calibrado con los estándares respectivos para cada parámetro de acuerdo con el National Institute of Standards and Technology (NIST). Para su determinación se emplearon reactivos de $\mathrm{NO}_{3}$ (Nitraver 5) y $\mathrm{NO}_{2}$ (Nitriver 2). El análisis de la alcalinidad se realizó en el laboratorio de Biotecnología Ambiental de El Colegio de la Frontera Sur (Unidad Tapachula), empleando una mezcla de reactivos indicadores (anaranjado de metilo y ácido sulfúrico, $95 \%$ a 98 $\%$ ), siguiendo el método la Norma Oficial Mexicana NOM-041-SSA1-1993 (SSA 1995). La evaluación bacteriológica del agua se llevó a cabo con la prueba Coliscan Easygel (BioPaddles), utilizando botellas con medio de cultivo de nutriente TTC agar y MacConkey agar. Las botellas se introdujeron en la muestra para obtener $40 \mathrm{~mL}$ de la misma, posteriormente se agitaron por $15 \mathrm{~s}$ y se vació el contenido para su posterior incubación a una temperatura de 35 $\pm 2{ }^{\circ} \mathrm{C}$ de 24 a $48 \mathrm{~h}$. Pasadas las primeras 24 y $48 \mathrm{~h}$ de incubación se realizaron el primero y segundo conteos de colonias, respectivamente (Tovar et al. 2013a). Los análisis bacteriológicos se hicieron por duplicado. La evaluación de $\mathrm{NO}_{3}, \mathrm{NO}_{2}$ y alcalinidad se realizaron por triplicado y directamente en campo. Debido a la técnica utilizada, los análisis de alcalinidad se hicieron en el citado laboratorio de Biotecnología. Dichos análisis se hicieron en los meses de mayo y abril de 2016.

\section{Análisis de datos}

Para el análisis de la información derivada de la entrevista se utilizó estadística descriptiva básica. Las diferencias entre sitios de los parámetros fisicoquímicos y bacteriológicos se determinaron a través de un análisis de varianza (ANDEVA). Se consideró una $\mathrm{p}$ $<0.05$ para reconocer diferencias significativas. El programa estadístico que se utilizó fue $\mathrm{R}$ v. 3.2.2. La evaluación de la calidad fisicoquímica y bacteriológica de las fuentes de agua se estimó a partir de las normas oficiales mexicanas NOM-041-SSA1-1993 (SSA 1995) y la NOM-127-SSA1-1994 (SSA 2000).

\section{RESULTADOS}

\section{Características de los entrevistados y las viviendas}

Se aplicó un total de 105 entrevistas domiciliarias (48 en isla de barrera y 57 en orilla del estero). Las entrevistas estuvieron representadas en su mayoría por mujeres en ambos estratos. En el estrato isla de barrera se entrevistó a un total de 34 mujeres y 14 hombres, mientras que en el estrato orilla del estero a un total de 33 mujeres y 23 hombres. La ocupación principal fue "ama de casa" en ambos estratos. En cuanto a educación, en promedio los sujetos cursaron el tercer grado de primaria para ambos sitios. La edad de los entrevistados varió de 45 a 60 años de edad, y han vivido en la comunidad de 35 a 50 años.

\section{Fuentes de abastecimiento de agua}

En el estrato isla de barrera todos los entrevistados expresaron tener pozo artesiano en su vivienda, y en algunos casos acceden a agua embotellada. En el sitio orilla del estero hay viviendas que cuentan con pozo artesiano, en tanto que otras acceden a agua embotellada y agua de la red.

El agua embotellada proviene de purificadoras de agua que se encuentran en comunidades vecinas y en la cabecera municipal. El costo del garrafón de $18 \mathrm{~L}$ varía por comunidad de 13 a 18 pesos. De acuerdo con los entrevistados, la venta de garrafones dañados y en malas condiciones es frecuente, continuamente contienen sedimentos y algas, tienen aberturas y son parchados con silicón.

En las dos comunidades que cuentan con agua entubada el agua proviene de pozos profundos que se encuentran en comunidades vecinas. El agua que se extrae llega a un tanque recolector ubicado en la comunidad; una vez almacenada, se realiza la cloración mediante el suministro de pastillas de cloro. Este proceso es realizado por el encargado en turno, quien no cuenta con capacitación para llevar a cabo 
dicho proceso. Cuando se carece de insumos para la cloración, el agua se suministra a las viviendas sin tratamiento previo.

\section{Determinación y percepción de la calidad del agua Isla de barrera}

La percepción de la calidad del agua del pozo es considerada de buena calidad por la mayoría de los entrevistados (Fig. 2). El principal uso es doméstico, en algunas viviendas la emplean para regar el patio o plantas y para aseo personal. Los principales factores que influyen en que el agua de los pozos sea de buena calidad, según los usuarios, son la vegetación, la filtración por la arena, la distancia de las viviendas respecto de las fosas sépticas, el estero y la ubicación en una isla de barrera. De acuerdo con los análisis de agua, la mayoría de los pozos no cumplen con los estándares de calidad establecidos por la NOM-127SSA1-1994 (SSA 2000) para consumo (Cuadro II). De los 16 pozos evaluados, 12 rebasaron los límites permitidos para $\mathrm{NO}_{2}(<0.05 \mathrm{mg} / \mathrm{L})$ y 11 para $\mathrm{NO}_{3}$ $(<10 \mathrm{mg} / \mathrm{L})$. En cuanto a la alcalinidad y el pH, ninguno rebasó lo establecido por la norma $(<300 \mathrm{mg} / \mathrm{L}$ y 8-5, respectivamente), pero se encuentran cerca de rebasarla. En 14 pozos se registró la presencia de Escherichia coli, en dos pozos de El Zapotal E. coli y Enterobacter sp., y en un pozo de El Palmarcito $E$. coli y Pseudomonas sp.

Quienes acceden a agua de garrafón, la consideran de buena calidad por ser más limpia y por su sabor más dulce en comparación con el agua de pozo. Otros la consideran de mala calidad por las condiciones de los garrafones y por el sedimento que tiene el agua (Fig. 2). Sin embargo, las cuatro marcas evaluadas

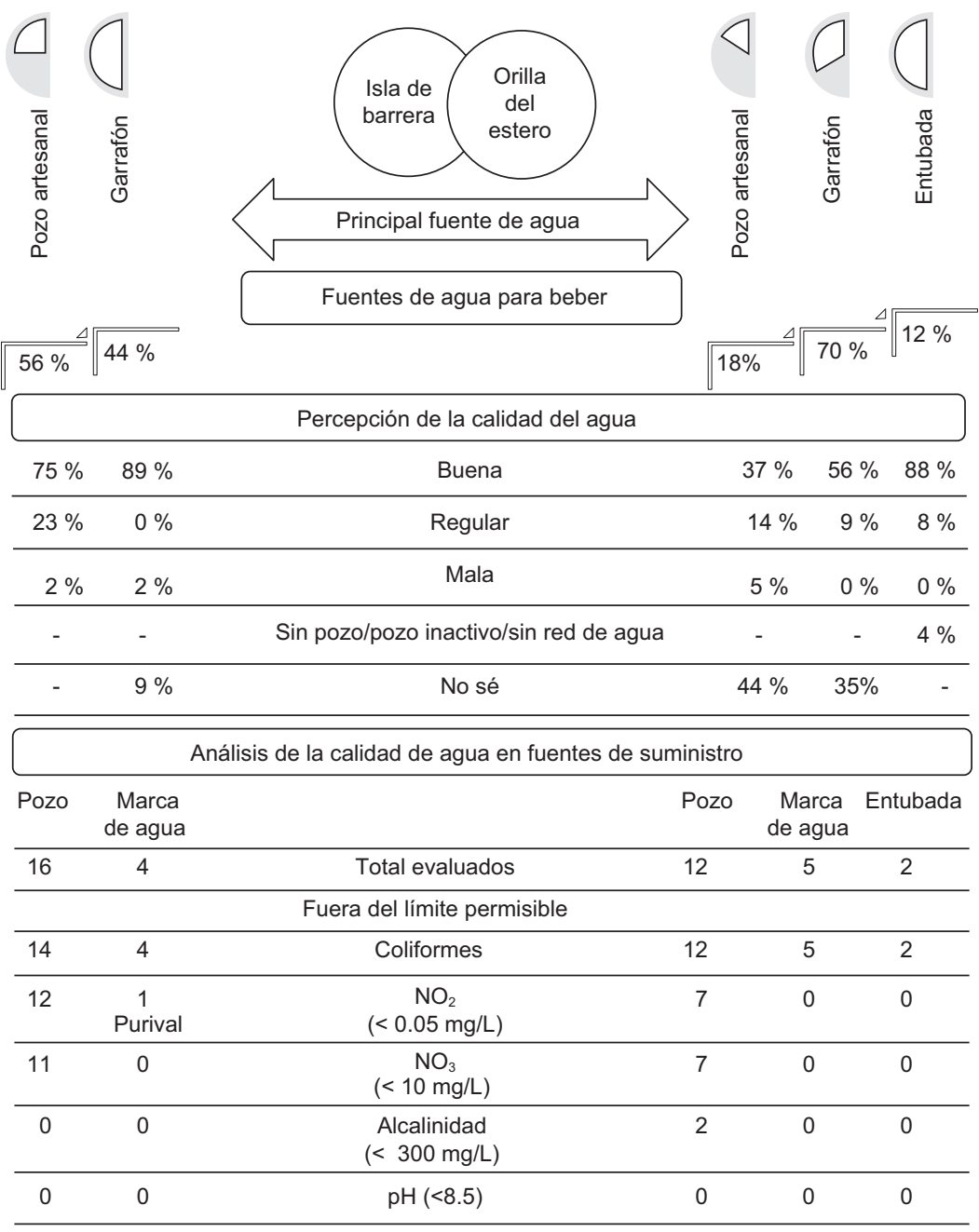

Fig. 2. Fuente principal de abastecimiento de agua, percepción de la calidad del agua y resultados del análisis del agua en las comunidades de estudio 


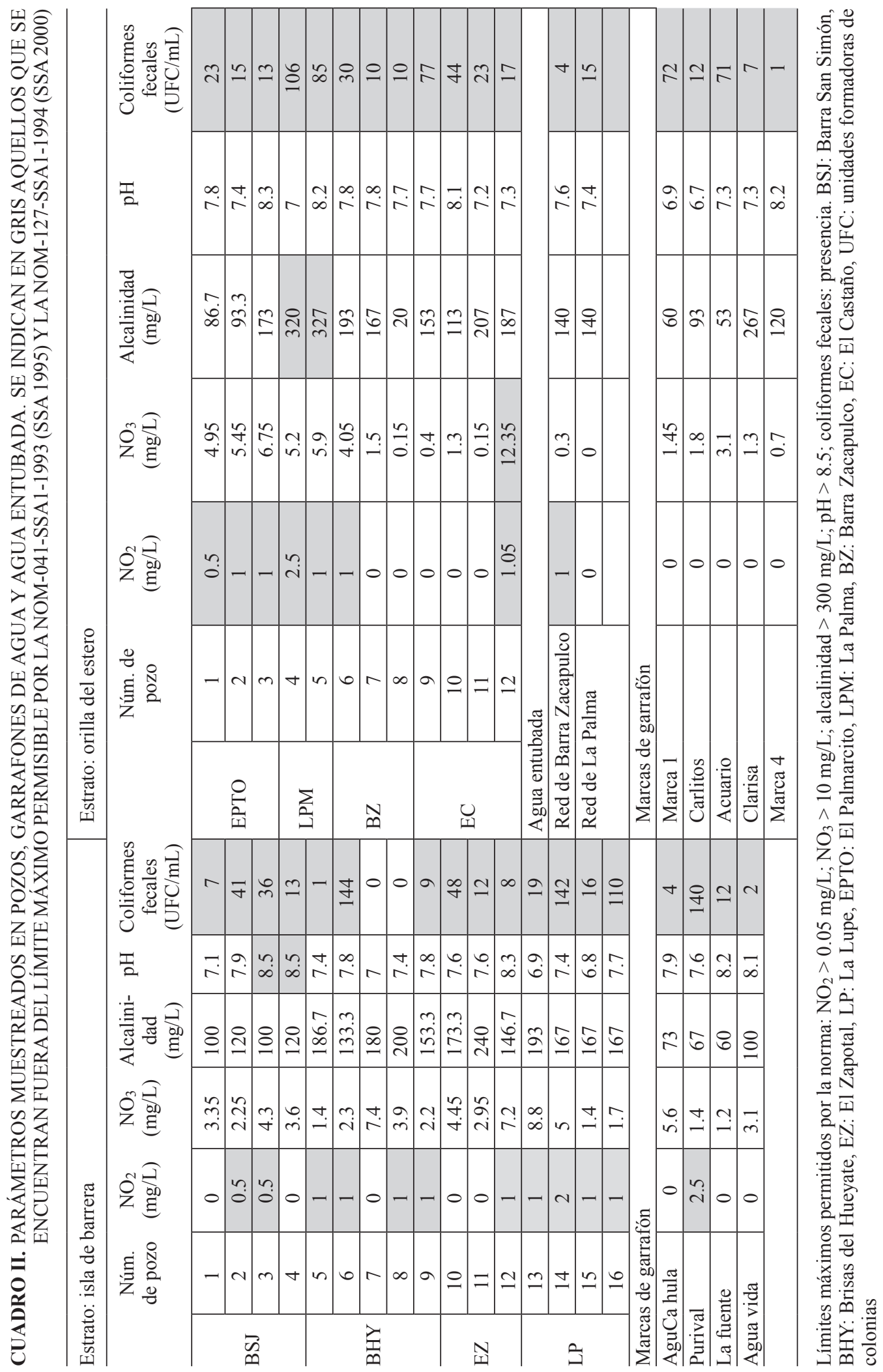


(Agua Chula, Purival, La Fuente, y Agua Vida) presentaron E. coli. Únicamente Purival rebasó el máximo permisible para $\mathrm{NO}_{2}(>0.05 \mathrm{mg} / \mathrm{L})$, en tanto que el resto se encontraron dentro de los límites permitidos para $\mathrm{NO}_{3}$, alcalinidad y $\mathrm{pH}(>10 \mathrm{mg} / \mathrm{L}$, $>300 \mathrm{mg} / \mathrm{L}$ y $>8.5 \mathrm{pH}$, respectivamente) (Fig. 2 y Cuadro II). Ninguna de las variables estudiadas presentó variación espacial (Cuadro III).

\section{Orilla del estero}

La mayoría de los entrevistados que poseen agua entubada la perciben de buena calidad en comparación con el agua de pozo (Fig. 2). El agua entubada se utiliza para regar plantas y patios de las casas, para consumo doméstico y para aseo personal. Los entrevistados asocian la calidad del agua de pozo principalmente con la filtración por la arena, la cercanía al estero, la distancia de su vivienda a la fosa séptica, la vegetación en su comunidad y la cercanía a la isla de barrera. Los análisis de calidad de agua mostraron que, de los 12 pozos evaluados, siete rebasan el máximo permisible para $\mathrm{NO}_{2}(>0.05 \mathrm{mg} / \mathrm{L})$ y para $\mathrm{NO}_{3}(>10 \mathrm{mg} / \mathrm{L})$. En cuanto a la alcalinidad, los pozos 4 y 5 rebasaron el máximo permitido ( $>$ $300 \mathrm{mg} / \mathrm{L}$ ), en tanto que ninguno rebasó el $\mathrm{pH}$ de 8.5 que establece la norma. Los 12 pozos presentaron coliformes fecales, especialmente E. coli (Fig. 2 y Cuadro II).

El agua embotellada es la principal fuente de agua para beber, y es considerada por la mayoría de los entrevistados como de buena calidad. Ninguna de las cinco aguas de garrafón disponibles (Carlitos, Acuario, Clarisa y dos sin marca) registró concentraciones de $\mathrm{NO}_{2}, \mathrm{NO}_{3}$, alcalinidad y $\mathrm{pH}$ mayores a lo que establece la norma, pero sí presentaron $E$. coli, por lo que se encuentran fuera de los establecido en la NOM-041-SSA1-1993 (SSA 1995). Ninguna variable presentó variación espacial (Cuadro III).

Las 24 viviendas que cuentan con agua entubada consideran que ésta es de buena o regular calidad. De estas mismas viviendas, el $44 \%$ no percibe la calidad del agua de pozo, y algunos entrevistados consideran que es de buena calidad (Fig. 2). El agua entubada es usada principalmente para aseo doméstico y para regar patio o plantas. Los datos que arrojan el análisis de agua para las dos redes de agua muestran qué

CUADRO III. ANÁLISIS DE VARIANZA (ANDEVA) ESPACIAL DE LAS VARIABLES MEDIDAS EN LOS ESTRATOS ISLA DE BARRERA Y ORILLA DEL ESTERO (VALORES PROMEDIO \pm DESVIACIÓN ESTÁNDAR)

\begin{tabular}{clccc}
\hline Variable & Estrato & Promedio & \pm Desviación estándar & ANDEVA espacial \\
\hline $\mathrm{O}_{2}$ & Isla de barrera & 2.2 & 1.2 & $\mathrm{P}=0.652$ \\
$(\mathrm{mg} / \mathrm{L})$ & Orilla del estero & 1.9 & 1.3 & \\
\hline $\mathrm{NO}_{2}$ & Isla de barrera & 0.8 & 0.5 & $\mathrm{P}=0.749$ \\
$(\mathrm{mg} / \mathrm{L})$ & Orilla del estero & 0.7 & 0.8 & $\mathrm{P}=0.911$ \\
\hline $\mathrm{NO}_{3}$ & Isla de barrera & 3.9 & 2.2 & \\
$(\mathrm{mg} / \mathrm{L})$ & Orilla del estero & 4 & 3.6 & $\mathrm{P}=0.577$ \\
\hline $\mathrm{Alcalinidad}$ & Isla de barrera & 155.8 & 39.8 & $\mathrm{P}=0.608$ \\
$(\mathrm{mg} / \mathrm{L})$ & Orilla del estero & 170 & 89.5 & $\mathrm{P}=0.151$ \\
\hline $\mathrm{CE}$ & Isla de barrera & 818.1 & 1360 & \\
$(\mu \mathrm{s} / \mathrm{cm})$ & Orilla del estero & 602.7 & 520.8 & $\mathrm{P}=0.644$ \\
\hline $\mathrm{Salinidad}$ & Isla de barrera & 3 & 4.8 & $\mathrm{P}=0.624$ \\
$(\mathrm{ppt})$ & Orilla del estero & 0.8 & 1.7 & $\mathrm{P}=0.994$ \\
\hline $\mathrm{pH}$ & Isla de barrera & 7.6 & 0.5 & \\
$(\mu \mathrm{s} / \mathrm{cm})$ & Orilla del estero & 7.7 & 0.4 & 2.1 \\
\hline $\mathrm{T}{ }^{\circ} \mathrm{C}$ & Isla de barrera & 30 & 2.5 & \\
\hline $\mathrm{Coliformes}$ & Isla de barrera & 37.9 & 33.1 & \\
$(\mathrm{UFC} / \mathrm{mL})$ & Orilla del estero & 37.8 & & \\
\hline & & & & \\
\hline
\end{tabular}

UFC: unidades formadoras de colonias 
aunque presentaron una densidad baja de coliformes (4 CFU/mL para la muestra de Barra Zacapulco y con $15 \mathrm{CFU} / \mathrm{mL}$ en la muestra de La Palma) ambas no son aptas para el consumo humano de acuerdo a la NOM041-SSA1-1993 (SSA 1995). Los valores de $\mathrm{NO}_{2}$ (1 y $0 \mathrm{mg} / \mathrm{L}), \mathrm{NO}_{3}(0.3$ y $0 \mathrm{mg} / \mathrm{L})$ y alcalinidad de $(140 \mathrm{mg} / \mathrm{L}$ en ambas) se encuentra por debajo del máximo permisible por la norma (Fig. 2 y Cuadro II). Ninguna de las variables presentó variación espacial (Cuadro III).

\section{Disponibilidad de agua en comunidades rurales como servicio ecosistémico}

Para los usuarios en el estrato isla de barrera la disponibilidad de agua en sus viviendas se reduce cuando las concentraciones de salinidad aumentan en el agua de pozo y provocan un cambio en el sabor que impide su consumo. La escasez de agua, especialmente en la temporada de estiaje, la contaminación de agua por fuentes externas, la limpieza y el mantenimiento de los pozos son otros factores que afectan la disponibilidad. Algunos entrevistados perciben una baja disponibilidad de agua pero no saben a qué atribuir este cambio, la cual es notoria de enero a mayo.

Algunos usuarios asentados en orilla del estero no perciben alteración en la disponibilidad del agua en los pozos, otros señalan que la salinidad y el mantenimiento de los pozos, así como la escasez de agua, son factores importantes. Los meses del año que identifican con mayor escasez de agua son marzo y abril.

Las personas que cuentan con agua entubada en las viviendas perciben escases en la disponibilidad del líquido debido a que el suministro se realiza cada tercer día.

La lluvia es considerada como un servicio ambiental. Aunque la gente no lo percibe como tal, si sabe que gracias a este fenómeno los pozos se recargan de agua; asimismo, percibe un aumento en la profundidad y, por ende, una disminución en la salinidad del agua que permite aprovecharla. También hay quienes advierten los beneficios en la pesca, con mayor producción, y en la realización de diversas actividades derivadas principalmente del agua de lluvia captada.

Para quienes dependen del agua entubada, el suministro es limitado debido a que la frecuencia de abastecimiento es de tres días por semana. Además de factores como la temporalidad, en este caso la temporada de lluvia acarrea fallas en la energía eléctrica, por ende obstáculos para el funcionamiento las bombas y la consecuente restricción en el suministro de agua. Pese a las situaciones que implica la temporada de lluvia, en algunos casos permite la captación de agua para realizar diversas actividades cuando falla el suministro de la misma (Fig. 3).

\begin{tabular}{|c|c|c|}
\hline \multicolumn{3}{|c|}{ Percepción de la alteración de la calidad de agua } \\
\hline $65 \%$ & Salinidad del agua & $36 \%$ \\
\hline $8 \%$ & Escasez de agua & $0 \%$ \\
\hline $2 \%$ & Contaminación & $0 \%$ \\
\hline $2 \%$ & Mantenimiento del pozo & $14 \%$ \\
\hline $23 \%$ & No sé & $50 \%$ \\
\hline \multicolumn{3}{|c|}{ Percepción de los beneficios de la lluvia en los pozos } \\
\hline $49 \%$ & Recarga de agua & $27 \%$ \\
\hline $32 \%$ & Aumento en la profundidad & $0 \%$ \\
\hline $32 \%$ & Disminución en la salinidad del agua & $25 \%$ \\
\hline $13 \%$ & Otros & $37 \%$ \\
\hline \multicolumn{3}{|c|}{$\begin{array}{l}\text { Meses con menor disponibilidad de agua } \\
\text { según la percepción de los usuarios }\end{array}$} \\
\hline $12 \%$ & Enero & $2 \%$ \\
\hline $12 \%$ & Febrero & $2 \%$ \\
\hline $19 \%$ & Marzo & $12 \%$ \\
\hline $25 \%$ & Abril & $20 \%$ \\
\hline $13 \%$ & Mayo & $2 \%$ \\
\hline $0 \%$ & Septiembre & $2 \%$ \\
\hline $2 \%$ & Noviembre & $0 \%$ \\
\hline $17 \%$ & No sé & $59 \%$ \\
\hline
\end{tabular}

Fig. 3. Percepción de la alteración en la disponibilidad de agua, beneficios de la lluvia y meses que escasea el agua

\section{DISCUSIÓN}

De acuerdo con la opinión de los entrevistados, la percepción de la calidad de agua determina los usos que se dan a las diversas fuentes del líquido; es decir, una fuente de agua que consideran de buena calidad es destinada para consumo doméstico, especialmente para beber en el caso del agua embotellada. Si el agua es considerada de calidad regular solamente se emplea para aseo personal y si es de mala calidad si 
únicamente sirve para regar el patio o las plantas. Dicha percepción puede atribuirse a que el mayor número de entrevistados fueron mujeres, ya que según Godínez y Lazos (2001) la percepción depende de la edad, el sexo y la experiencia. El rol de las mujeres en las comunidades, por ejemplo, en el desempeño de diversas actividades domésticas, les permite establecer parámetros de la calidad del agua definidos por su experiencia y el uso que le dan a las diferentes fuentes de este recurso. Gutiérrez-Villalpando et al. (2013) señalan que las mujeres son las principales usuarias del agua para el consumo doméstico, la agricultura de subsistencia, la salud y el saneamiento, por lo que perciben mejor los cambios en torno al líquido.

Los análisis bacteriológicos del agua de pozo y el agua entubada mostraron un recurso apto para uso doméstico, pero no para beber. Aunque dentro de los usos que le dan los entrevistados no está este último, debe considerarse una forma de proveer a la población agua apta para el consumo humano. Por su parte, los valores registrados del agua potable embotellada representan un riesgo para la salud, ya que la presencia de coliformes fecales evidencian un líquido no garantizado para beberse.

Los análisis fisicoquímicos y bacteriológicos del agua de pozo y entubada demostraron contaminación por coliformes fecales. Las concentraciones de $\mathrm{NO}_{2}$, $\mathrm{NO}_{3}$, alcalinidad y $\mathrm{pH}$ en algunos pozos rebasaron el límite máximo permisible y en otros están cerca de rebasarlo. De acuerdo con la NOM-127-SSA1-1994, el consumo de las fuentes de agua analizadas es un riesgo para la salud por que no es adecuada ni apta para este fin (SSA 2000).

De acuerdo con la percepción de los usuarios, el agua potable embotellada es la fuente principal de agua para beber, debido a que es considerada como de mejor calidad en comparación con el agua de pozo y entubada. Según algunos estudios, la adquisición de agua embotellada en los hogares es una forma de "comportamiento defensivo", porque los usuarios perciben la relación entre calidad del agua y salud (Barreiro-Hurlé y Pérez y Pérez 2006, Benez 2008) y consideran que el agua embotellada tiene menos impurezas (Ferrier 2001, Bohórquez 2007); sin embargo, esto no garantiza el consumo de agua de mejor calidad, ya que ésta no cumple con los estándares de calidad establecidos por la NOM-041-SSA1-1993 para agua envasada y purificada (SSA 1995). En estudios previos sobre calidad del agua embotellada, los usuarios manifestaron que también necesita tener un tratamiento previo al consumo, puesto que sobrepasa lo establecido por las normas respectivas (Robles et al. 1999, Mazari-Hiriart et al. 2000, Díaz et al. 2007,
Ahmed et al. 2013, Monteverde et al. 2013). Rojas (2010) indica que el consumo de agua contaminada con coliformes predispone a los usuarios a desarrollar sintomatología relacionada con trastornos como enfermedad diarreica aguda (EDA) y hepatitis A.

Los usuarios perciben una limitada disponibilidad de agua en sus fuentes de suministro, especialmente en las viviendas que tienen acceso al agua entubada, y también que los problemas en el suministro de la red de agua son infrecuentes. Autores como Chávez et al. (2013) y Soares y Vargas (2008) mencionan que los pozos son una alternativa en zonas rurales que no tienen acceso a sistemas de agua potable o los sistemas son deficientes. Soares (2007) y Benez et al. (2010) mencionan que la inaccesibilidad al servicio básico de agua potable en las zonas rurales depende de muchos factores, entre ellos la localización de la fuente de abastecimiento, al igual que las características del propio entorno físico (Fernández-Moreno 2008) y el tamaño de la población (Lazos y Paré 2000). A los beneficios que provee el uso de pozos artesianos, hay que sumarles los bienes que proporciona el entorno natural en las dunas costeras. Aunque los entrevistados no reconocen como tales los servicios ambientales de las dunas, si perciben los diversos beneficios que la lluvia aporta a los pozos, aprovechando mejor el líquido en la temporada de lluvia.

Dentro de los múltiples beneficios de las dunas costeras se encuentra la purificación del agua y como resultado su calidad (Carrera et al. 2016); sin embargo, los valores de contaminación de las fuentes de agua analizadas son altos debido a una gran influencia de procesos antrópicos. Por ejemplo, la contaminación del agua de los ríos que desembocan en La Encrucijada, tal como lo muestra la evaluación de la calidad de agua de siete ríos que desembocan en la reserva. En dicha evaluación se consideraron tanto las temporadas de secas como de lluvias, y en ambas se registraron valores altos de coliformes fecales por arriba de 500 CFU/100 mL (Tovar et al. 2013b).

La contaminación del agua entubada puede estar influenciada por las formas de tratamiento y el déficit de preparación de los encargados de realizar los procesos. Haro et al. (2012) enfatizan en su estudio la necesidad de vigilar los procesos de cloración del agua, especialmente en la red pública. En el estudio realizado por Torres et al. (2009) se evidencia que la causa principal del suministro de agua no apta para consumo es la incapacidad de los prestadores del servicio del acueducto para sanear el agua.

La contaminación del agua embotellada puede deberse a la contaminación en el proceso de purificación y a las condiciones de los garrafones de agua. 


\section{CONCLUSIONES}

De acuerdo con la opinión de los entrevistados, la percepción de calidad del agua de las diferentes fuentes determina el uso que se les da. En cuanto a los parámetros bacteriológicos del agua de pozo y entubada durante el periodo de estudio, ésta no fue apta para consumo humano según la normatividad nacional.

Los parámetros bacteriológicos del agua embotellada que se comercializó en las comunidades durante el periodo de estudio indican asimismo que no es apta para consumo humano de acuerdo con la normatividad nacional. Los parámetros fisicoquímicos evaluados para las muestras de agua de pozos y entubada están cerca de rebasar el límite máximo permisible establecido por la Norma Oficial Mexicana NOM-127-SSA-1994 (SSA 2000) para agua de uso y consumo humano, en tanto que los parámetros fisicoquímicos del agua embotellada todavía se encuentran dentro del rango permitido.

La información derivada de este estudio debería considerarse como elemento importante para los planeadores y manejadores del área natural protegida La Encrucijada y para las autoridades encargadas de la gestión del agua en los diversos niveles de intervención.

\section{AGRADECIMIENTOS}

Se agradece al Consejo Nacional de Ciencia y Tecnología (CONACyT) por la beca número 662298 otorgada para la Maestría en Ciencias de Recursos Naturales y Desarrollo Rural. Al equipo de la Comisión Nacional de Áreas Naturales Protegidas (CONANP) Reserva de la Biósfera La Encrucijada, Chiapas, por brindar todas las facilidades para la realización de este proyecto. A los habitantes de las localidades Barra San José, Brisas de Hueyate, La Palma, La Lupe, Barra Zacapulco, El Castaño, El Zapotal y El Palmarcito, por su hospitalidad y facilidades para realizar el trabajo de campo. Al Laboratorio de Biotecnología Ambiental de El Colegio de la Frontera Sur, Unidad Tapachula, por el espacio brindado para la realización de los análisis correspondientes de este trabajo y por el préstamo del equipo $\mathrm{HACH}$ para el análisis de muestras en campo. A la M.C. María Guadalupe Albarrán Rivas y a los ingenieros Antonio de Jesús Yáñez Pérez Edder R. Monzón de Cruz por la ayuda proporcionada en campo para la realización de las entrevistas y la toma de muestras.

\section{REFERENCIAS}

Ahmed W., Yusuf R., Hasan I., Ashraf W., Goonetilleke A., Toze S. y Gardner T. (2013). Fecal indicators and bacterial pathogens in bottled water from Dhaka, Bangladesh. Braz. J. Microbiol. 44 (1), 97-103. DOI: $10.1590 / \mathrm{S} 1517-83822013005000026$

Álvarez-Salas L.M., Gómez-Aguirre A.M. y Cano-López W.A. (2016). Percepciones de los servicios ecosistémicos en el complejo de páramos Frontino-Urrao, Departamento de Antioquia, Colombia. Biota Colombiana 17 (2), 134-147.

DOI: $10.21068 / \mathrm{C} 2016 \mathrm{v} 17 \mathrm{~s} 02 \mathrm{a} 08$

Arias C.C.A. (2006). Enfoques teóricos sobre la percepción que tienen las personas. Horizontes Pedagógicos 8 (1), 9-22.

Arellano E., Camarena L., von Glascoe C. y Daesslé W. (2009). Percepción del riesgo en salud por exposición a mezclas de contaminantes: el caso de los valles agrícolas de Mexicali y San Quintín, Baja California, México. Revista Facultad Nacional de Salud Pública 27 (3), 291-301.

Barreiro-Hurlé J. y Pérez y Pérez L. (2006). Beneficios sociales de la mejora en la calidad del agua; una aproximación a partir de los costes defensivos en los hogares. Estudios de Economía Aplicada 24 (1), 453-476.

Barrios-Ramos M.A. (2013). Cambios en la línea de costa del municipio de Tapachula, Chiapas, México. Tesis de Licenciatura. Centro de Biociencias, Universidad Autónoma de Chiapas, México, 33 pp.

Bohórquez A.L. (2007). Crisis del agua, biopolítica y desarrollo humano. Management 16 (28), 125-145.

Benez M.C. (2008). Percepciones de la calidad y de la gestión del agua de las aguas superficiales de la cuenca de San Cristóbal de Las Casas, Chiapas, México. Tesis de Maestría. El Colegio de la Frontera Sur, San Cristóbal de las Casas, Chiapas, México, 146 pp.

Benez M.C., Kauffer M.E.F. y Álvarez G.G. del C. (2010). Percepciones ambientales de la calidad del agua superficial en la microcuenca del río Fogótico, Chiapas. Front. Norte 22 (23), 129-158.

Calixto F.R. y Herrera R.L. (2010). Estudio sobre las percepciones y la educación ambiental. Tiempo de Educar 11 (22), 227-249.

Camacho-Valdez V., Ruiz-Luna A., Ghermandi A. y Nunes P.A.L.D. (2013). Valuation of ecosystem services provided by coastal wetlands in northwest Mexico. Ocean. Coast. Manage. 78, 1-11. DOI: 10.1016/j.ocecoaman.2013.02.017

CARE (2012). Programa Unificado de Fortalecimiento de Capacidades. Módulo 8: Gestión integrada del recurso hídrico. CARE Internacional-Avina. Informe. Quito, Ecuador, $181 \mathrm{pp}$. 
Carrera H.J.J., Peralta P.L.A. y Sánchez H.L.E. (2016). La filtración y depuración del agua. En: Servicios ecosistémicos de las selvas y bosques costeros de Veracruz. (Moreno-Casasola P., Ed.). INECOL-ITTOCONAFOR-INECC, Xalapa, Veracruz, México, pp. 143-157.

Chávez V.M., Rivera H.R., Romero C.T. y Vizcarra B.I. (2013). El pozo: usos, seguridad y tradición en la subcuenca del río San Javier. Estudios Sociales 21 (41), 262-286.

Cejudo-Espinosa E., Ramos-Valdivia A.C., Esparza-García F., Moreno-Casasola P. y Rodríguez-Vázquez R. (2008). Short-term accumulation of Atrazine by three plants from a wetland model system. Arch. Environ. Con. Tox. 56 (2), 201-208.

DOI: $10.1007 / \mathrm{s} 00244-008-9193-7$

Cochran W. (2000). Técnicas de muestreo. 15 ed. CECSA, México, $513 \mathrm{pp}$.

CONAGUA (2016). Situación del subsector agua potable, drenaje y saneamiento. Subdirección General de Agua Potable, Drenaje y Saneamiento, Comisión Nacional del Agua, Ciudad de México, 96 pp.

CONAPO (2015). Situación demográfica de México. Base de datos. Comisión Nacional de Población, México, 308 pp.

Corral-Verdugo V., Tapia F.C., Fraijo S.B., Mireles A.J. y Márquez U.P. (2008). Orientación a la sustentabilidad como determinante de los estilos de vida sustentables: un estudio con una muestra mexicana. Rev. Mex. Psicol. 25 (2), 313-327.

Díaz J.C., Caraballo H., Villareal M., Lobo H., Rosario J., Briceño J., Gutiérrez G. y Díaz S. (2007). Is bottled water adapted for our consumption? Acad. VI (11), 2-12.

Everard M., Jones L. y Watts B. (2010). Have we neglected the societal importance of sand dunes? An ecosystem services perspective. Aquat. Conserv. 20 (4), 476-487. DOI: $10.1002 /$ aqc. 1114

Fisher J. y Acreman M. (2004). Wetland nutrient removal. A review of the evidence. Hydrol. Earth Syst. Sc. 8 (4), 673-685. DOI: 10.5194/hess-8-673-2004

Fernández-Moreno Y. (2008). ¿Por qué estudiar las percepciones ambientales? Una revisión de la literatura mexicana con énfasis en Áreas Naturales Protegidas. Espiral Estudios sobre Estado y Sociedad 15 (43), 179-202.

Ferrier C. (2001). Bottled water: understanding a social phenomenon. AMBIO 30 (2), 118-119.

DOI: $10.1579 / 0044-7447-30.2 .118$

Gómez-Ortega R. (2013). Ecología del sistema lagunar Chantuto-Panzacola, Chiapas, basada en la aplicación e interpretación de algunos índices tróficos, parámetros físico-químicos y biológicos. Tesis de Maestría.
El Colegio de la Frontera Sur, Tapachula, Chiapas, México, 109 pp.

Godínez L. y Lazos E. (2001). Percepciones y sentires de las mujeres sobre el deterioro ambiental: retos para su empoderamiento. En: Género y medio ambiente (Tuñón E., Ed.). El Colegio de la Frontera Sur-Secretaria de Medio Ambiente y Recursos Naturales-Plaza y Valdés. México, pp. 145-177.

González-Figueroa R., Gerritsen P.R.W. y Malischke T.K. (2007). Percepciones sobre la degradación ambiental de agricultores orgánicos y convencionales en el ejido La Ciénega, municipio de El Limón, Jalisco, México. Economía, Sociedad y Territorio 7 (25), 215-239.

Gutiérrez-Villalpando V., Nazar-Beutelspacher D., ZapataMartelo E., Contreras-Utrera J., y Salvatierra-Izaba B. (2013). Mujeres y organización social en la gestión del agua para consumo humano y uso doméstico en Berriozábal, Chiapas. LiminaR 11 (2), 100-113.

DOI: 10.29043/liminar.v11i2.225

Haro J.A., Nubes G. y Ortiz R.C.O. (2012). Riesgos sanitarios en calidad bacteriológica del agua. Una evaluación en diez estados de la república mexicana. Región y Sociedad 24 (3), 257-288.

Hernández H.J.L. (2014). Caracterización de uso de suelo y evaluación de la calidad riparia del río Cacaluta, Acacoyagua, Chiapas, México. Tesis de Maestría. El Colegio de la Frontera Sur, Tapachula, Chiapas, México, $180 \mathrm{pp}$.

INEGI (2010). Base de datos del censo de población y vivienda 2010. Instituto Nacional de Estadística y Geografía [en línea]. http://www3.inegi.org.mx/sistemas/tabuladosbasicos/tabentidad.aspx?c=33713\&s $=$ est $15 / 01 / 2015$

INEGI (2015). Base de datos del censo de población y vivienda 2015. Instituto Nacional de Estadística y Geografía [en línea]. http://www.beta.inegi.org.mx/proyectos/enchogares/especiales/intercensal/ 15/01/2015

Jones S.A. y Mach P.B. (2012). Pesca responsable: percepciones locales, sobre las pesquerías artesanales de La Reserva de la Biósfera La Encrucijada, Chiapas, México. Tesis de Maestría. El Colegio de la Frontera Sur, Tapachula, Chiapas, México, 146 pp.

Kauffer M. (2014). De las cuencas a la gestión integrada de los recursos hídricos (GIRH) en Chiapas: una construcción histórica plasmada en escenarios concretos. En: Cuencas en Chiapas: la construcción de utopías en cascada (Kauffer M., Ed.). Centro de Investigaciones y Estudios Superiores en Antropología Social, Chiapas, México, pp. 17-51.

Lazos E. y Paré L. (2000). Miradas indígenas sobre una naturaleza entristecida. Percepciones del deterioro ambiental entre nahuas del sur de Veracruz. Plaza y Valdés-Universidad Autónoma de México-Instituto de Investigaciones Sociales, México, 220 pp. 
Liscovsky I.J., Arce-Ibarra A.M., Parra-Vázquez M.R. y Bello-Baltazar E. (2012). Discurso territorial y práctica sectorial de las políticas socioambientales: un análisis del ecoturismo. Economía, Sociedad y Territorio 12 (39), 359-402.

Machuca J.M.A. (2014). Diagnóstico del estado actual del uso y manejo de la zona costera en el Estado de Chiapas. Tesis de Maestría. El Colegio de la Frontera Sur, Tapachula, Chipas, México, 194 pp.

Martínez-Carazo P.C. (2006). El método de estudio de caso Estrategia metodológica de investigación científica. Pensamiento y Gestión (20), 165-193.

Mazari-Hiriart M., Cifuentes E., Velázquez E. y Calva J.J. (2000). Microbiological groundwater quality and health indicators in Mexico City. Urban. Ecosyst. 4 (2), 91-103. DOI: 10.1023/A:1011334326405

Monteverde M., Cipponeri M., Angelaccio C. y Gianuzzi L. (2013). Origen y calidad del agua para consumo humano: salud de la población residente en el área de la cuenca Matanza-Riachuelo del Gran Buenos Aires. Salud Colect. 9 (1), 53-63.

Moreno-Casasola P. (2010). Veracruz. Mar de arena. Comisión Organizadora del Estado de Veracruz de Ignacio de la Llave para la Conmemoración del Bicentenario de la Independencia Nacional y del Centenario de la Revolución Mexicana. Secretaría de Educación, Gobierno del Estado de Veracruz, Xalapa, Veracruz, México, 283 pp.

Mollema P.N., Antonellini M., Minchio A. y Gabbianelli G. (2008). The Influence of three-dimensional dune topography on salt-water intrusion in Marina Romea, Italy: A numerical modeling study using LIDAR data. Memorias. 20th Saltwater Intrusion Meeting. Naples, Florida, 23-27 de junio.

Pérez B.C.D., Gómez G.C.M. y del Villar G.A. (2011). El riesgo de disponibilidad de agua en la agricultura: una aplicación a las cuencas del Guadalquivir y del Segura. Estudios de Economía Aplicada 29 (1), 333-357.

Pimienta-Lastra R. de J. (1999). Esquemas de muestreo y márgenes de confiabilidad en encuestas de opinión política. Sociológica 14 (39), 183-202.

Pimienta-Lastra R. de J. (2000). Encuestas probabilísticas vs. no probabilísticas. Política y Cultura (13), 263-276.

Robles E., Ramírez P., González M.E., Sainz M.G., Martínez B., Durán A. y Martínez M.E. (1999). Bottledwater quality in metropolitan Mexico City. Water Air Soil Pollut. 113 (1-4), 217-226.

DOI: 10.1023/A:100508801
Rojas A. (2010). Determinación de coliformes totales, coliformes fecales y Vibrio sp. en aguas de consumo humano, provenientes de pozos y plantas de tratamiento del municipio San Fernando, estado Apure. Tesis de Licenciatura. Escuela de Ciencias, Universidad de Oriente, Anzoátegui, Venezuela, 51 pp.

Sánchez-Pérez H., Vargas-Morales M. y Méndez-Sánchez J. (2000). Calidad bacteriológica del agua para consumo humano en zonas de alta marginación de Chiapas. Salud Pública México 42 (5), 397-406.

Soares D. (2007). Acceso, abasto y control del agua en una comunidad indígena Chamula en Chiapas: Un análisis a través de la perspectiva de género, ambiente y desarrollo. Región y Sociedad 19 (38), 25-50.

Soares D. y Vargas V.S. (2008). El debate actual: entre economía, el derecho humano y la sustentabilidad. En: La gestión de los recursos hídricos: realidades y perspectivas. Tomo 1. (Soares D., Vargas S. y Nuño M.R., Eds.). Instituto Mexicano de Tecnología del Agua. Jiutepec, Morelos, México, pp. 87-119.

SSA (1995). Norma Oficial Mexicana NOM-041SSA1-1993. Bienes y servicios. Agua purificada envasada. Especificaciones sanitarias. Secretaría de Salud. Diario de Oficial de la Federación, 24 de marzo.

SSA (2000). Norma Oficial Mexicana NOM-127SSA1-1994. Salud ambiental. Agua para uso y consumo humano. Límites permisibles de calidad y tratamientos a que debe someterse el agua para su potabilización. Secretaría de Salud. Diario de Oficial de la Federación, 22 de noviembre.

Torres P., Hernán Cruz C. y Patiño P.J. (2009). Índices de calidad de agua en fuentes superficiales utilizadas en la producción de agua para consumo humano. Una revisión crítica. Revista Ingenierías Universidad de Medellín 8 (15), 79-94.

Tovar J.E., Calderón-Mandujano R.R., Espinoza T.A. y Rodríguez G.J. (2013a). Técnicas de muestreo y evaluación de servicios ecosistémicos y biodiversidad para la zona sierra y costa de Chiapas, México. Proyecto Ecosechas, manual del usuario. Centro de Biociencias, Universidad Autónoma de Chiapas-Conservación Internacional México, Tapachula, Chiapas, México, 61 pp.

Tovar J.E., Calderón-Mandujano R.R., Espinoza T.A. y Rodríguez G.J. (2013b). Monitoreo en plan de prueba de indicadores clave de servicios ecosistémicos (SECaptura de carbono, calidad de agua, calidad del suelo), biodiversidad (BD) y usos del suelo (Uds) en la Sierra Madre y costa de Chiapas. Informe final. Centro de Biociencias, Universidad Autónoma de Chiapas, Tapachula, Chiapas, México, 41 pp. 


\section{APÉNDICE 1. ENTREVISTA}

LP/EN; ${ }^{1} \mathrm{EZ} / \mathrm{EN} ;{ }^{2} \mathrm{BSJ} / \mathrm{EN} ;{ }^{3} \mathrm{BHY} / \mathrm{EN} ;{ }^{4} \mathrm{LPM} / \mathrm{EN} ;{ }^{5} \mathrm{BZ} / \mathrm{EN} ;{ }^{6} \mathrm{EC} / \mathrm{EN} ;{ }^{7} \mathrm{EPTO} / \mathrm{EN}^{8}$

Fecha__ I__ /2016 Nombre:

Sexo:

a) Hombre

b) Mujer

Edad:

18-30

$30-45$

45- 60

¿Cuántos integrantes son en la vivienda?

$2-4$

4-6

6-8

Tiene algún cargo en la comunidad:

Sí

No

Cuál:

Es originario de la comunidad:

Sí

No

Años de vivir en la comunidad

$10-15$

$15-25$

25-35

$35-50$

Escolaridad:

Analfabeta

Educación básica (primaria y secundaria)

Educación media superior y superior

¿Ocupación actual?

Labores agrícolas

Comerciante

Ama de casa

Pescador

Estudiante

Otro___ Especifique

Suministro de agua de pozo

1. El agua que bebe, proviene de:

Pozo

Agua de garrafón

Agua entubada

${ }^{1}$ La Lupe; EN: número de encuesta

${ }^{2}$ El Zapotal; EN: número de encuesta

${ }^{3}$ Barra San José; EN: número de encuesta

${ }^{4} \mathrm{El}$ Hueyate; EN: número de encuesta

${ }^{5}$ La Palma; EN: número de encuesta

${ }^{6}$ Barra Zacapulco; EN: número de encuesta

${ }^{7}$ El Castaño; EN: número de encuesta

${ }^{8}$ El Palmarcito; EN: número de encuesta 
2. ¿El pozo que usted tiene está activo?

Sí

No

No tengo

3. ¿Qué otro uso le da al agua de pozo?

Aseo personal

Aseo doméstico

Cocinar

Otro

Todos

4. ¿Cómo considera la calidad de agua de su pozo?

Buena ${ }^{1}$

Regular $^{2}$

Mala $^{3}$

5. De las Siguientes opciones solamente elija una, ¿considera usted, que la calidad del agua de pozo se encuentra asociado con?

Vegetación

Cercanía de la Isla

La arena ayuda a filtrar el agua

Lejos de la fosa séptica

Cercanía del estero

Otros

6. De las siguientes opciones solamente elija una, ¿cuál ha sido para usted un problema asociado al pozo?

Mantenimiento

Contaminación del agua

Escasez de agua

Salinidad del agua

Otras

7. ¿Mantiene tapado él pozo?

Sí

No

8. Su pozo es:

Artesiano (cemento o block)

Profundo (PVC): profundidad

9. ¿Tiene permiso por el pozo?

Sí

No

Agua de entubada

10. ¿Cuenta con el servicio de agua entubada en su vivienda?

Sí

No

11. ¿Cuántas veces a la semana llega el agua entubada a su vivienda?

Una vez a la semana

Dos veces a la semana

Tres veces a la semana

Todos los días

12. ¿Cómo considera qué es la calidad de agua de la tubería?

Buena

\footnotetext{
${ }^{1}$ Agua sin sedimento, sin olor, no está salada

${ }^{2}$ El agua tiene mucho sedimento, mal olor, sabor a sal, pero sirve para otras actividades no necesariamente para tomarla

${ }^{3}$ Agua con mucho sedimento y coloración amarilla, olor a huevo podrido o lodo, sabor salado
} 


\section{Mala}

No sé

13. ¿Aproximadamente, cuánto paga al mes por el agua entubada?

$\$ 20.00$ o más

\$ 30.00 o más

$\$ 50.00$ o más

Otro

14. ¿Considera que el precio que paga por el servicio de agua entubada es?

Muy caro

Caro

Adecuado

Barato

15. ¿Cuándo se presenta un problema con la tubería de agua en la comunidad, quién se encarga de solucionarlo?

El comité

El municipio

La comunidad y municipio

El comité y los usuarios

Todos

Agua de garrafón

16. De las siguientes opciones solamente elija una, ¿cuál considera que es la causa por la que usted compra garrafón de agua?

El agua de pozo esta salada

Porque los demás compran garrafón de agua

El agua es limpia

No tengo pozo

Otra

17. Aproximadamente, ¿cuánto paga por un garrafón de agua?

$\$ 12.00$ o más

$\$ 15.00$ o más

$\$ 18.00$ o más

$\$ 21.00$ o más

No sé

18. Aproximadamente, ¿cuántos garrafones de agua compra a la semana?

1 garrafón

2 garrafones

3 garrafones o más

Todos los días

No compro

No sé

Problemas de salud

19. De las siguientes opciones, elija solamente el síntoma más frecuente que ha presentado algún integrante de su familia:

Diarrea

Dolor de estómago

Vómito

Dolor de cabeza

Fiebre

Dengue

Zika

Chinkungunya

Ninguno

Otro Especifique: 
20. ¿Usted cree que la molestia que señaló anteriormente está relacionada con el agua que consume?

Sí

No

No sé

Disponibilidad de agua

21. ¿En qué mes del año ha notado que disminuye la profundidad del pozo?

\begin{tabular}{|c|c|c|c|c|c|c|c|c|c|c|c|}
\hline Ene & Feb & Mar & Abr & May & Jun & Jul & Ago & Sep & Oct & Nov & Dic \\
\hline
\end{tabular}

22. De las siguientes opciones elija solamente una, ¿cuál es el beneficio más importante que le brinda la lluvia?

Se recargan los pozos y aumenta la profundidad

Utilizamos agua de lluvia para realizar algunas actividades (uso doméstico, uso personal)

El agua de pozo es más dulce

Otro

Sobre las responsabilidades y organización comunitaria

23. ¿Existe comité de agua en la comunidad?

Sí

No

No sé

24. ¿Quiénes conforman el comité de agua?

Mujeres

Hombres

Ambos

No sé

25. ¿Cómo elijen a los integrantes del comité de agua?

Votación

Obligados

Disponibilidad de la persona

Por lista de nombres

No sé

26. ¿Sí le dieran la oportunidad de integrarse al comité de agua, estaría dispuesto a participar?

Sí

No

27. ¿Sabe cuáles son las funciones del comité de agua?

Gestionar agua a la comunidad (gestionar agua entubada ante cualquier dependencia)

Mantener el agua en la comunidad (administrar, vigilar, cobrar cuotas, organizar asambleas, etc.)

No sé

28. ¿Sabe cuándo se reúnen para las asambleas del agua?

Cada mes

Cada dos meses

Cuando se presente asuntos importantes

No sé

Otro

29. ¿Quiénes asisten a la asamblea del agua?

Usuarios

Avecindados

Todos en la comunidad

No sé

30. ¿Los usuarios del agua operan bajo un reglamento?

Sí

No

No sé 
31. ¿Usted a quien considera responsable para tratar los asuntos relacionados al agua en la comunidad?

A la comunidad (usuarios)

La cabecera municipal

A CONAGUA

No sé

Todos

32. ¿En la comunidad existen un grupo de personas que se dedican a evaluar la calidad de agua en las viviendas? Sí

No

No sé

33. ¿Sí le dieran la oportunidad de participar como miembro de un equipo donde su función de evaluar la calidad de agua en las viviendas, usted participaría?

Sí

No

No sé

34. Cree usted, que el tiempo que destinan para tratar los asuntos del agua es suficiente:

Sí

No

Según el asunto

No sé

Disposición de aguas servidas

35. ¿Su vivienda cuenta con fosa séptica?

Sí

No

No sé

36. ¿Dónde depositan el agua que se genera de lavar ropa y trastes?

En la fosa séptica

Se vierte al suelo y plantas

Se vierte al estero

Disposición de tener agua entubada

37. De las siguientes opciones solamente elija una, ¿cuál cree que es la razón por la que el agua entubada no llega a su comunidad?

Es difícil el acceso a la comunidad

No se ha gestionado el agua entubada

La comunidad no está dispuesta a pagar agua entubada

Otra

38. ¿Sí tuviera la oportunidad de tener agua entubada, lo pagaría?

Sí

No

No sé

39. Aproximadamente, ¿cuánto está dispuesto a pagar mensualmente?

\$20.00 o más

\$ 30.00 o más

$\$ 40.00$ o más

$\$ 50.00$ o más

No sé

Ninguna de las anteriores

40. ¿Sí tuviera agua entubada seguiría cuidando su pozo?

No

Sí

No sé 